\title{
Study of photon dominated regions in IC 348
}

\section{K. Sun ${ }^{1}$, C. Kramer ${ }^{1}$, B. Mookerjea ${ }^{2}$, V. Ossenkopf ${ }^{1,3}$, M. Röllig ${ }^{1,4}$ and J. Stutzki ${ }^{1}$}

${ }^{1}$ I. Physikalisches Institut, Universität zu Köln, Germany ${ }^{2}$ Department of Astronomy, University of Maryland, USA ${ }^{3}$ SRON National Institut for Space Research, the Netherlands ${ }^{4}$ Argelander-Institut für Astronomie, Universität Bonn, Germany

\begin{abstract}
We present fine structure line of neutral carbon at $492 \mathrm{GHz}\left({ }^{3} \mathrm{P}_{1}-{ }^{3} \mathrm{P}_{0}\right.$, hereafter [C I] 1-0) and ${ }^{12} \mathrm{CO} 4-3$ KOSMA observations. This data has been combined with FCRAO ${ }^{12} \mathrm{CO} 1-0$ and ${ }^{13} \mathrm{CO} 1-0$ data. We have used these observations to understand the emission from the photon dominated regions (PRDs) in IC 348. We confirm the anti-correlation between $\mathrm{N}(\mathrm{C}) / \mathrm{N}(\mathrm{CO})$ and $\mathrm{N}\left(\mathrm{H}_{2}\right)$ as seen in most Galactic PDRs (Mookerjea et al. 2006).
\end{abstract}

\section{Results}

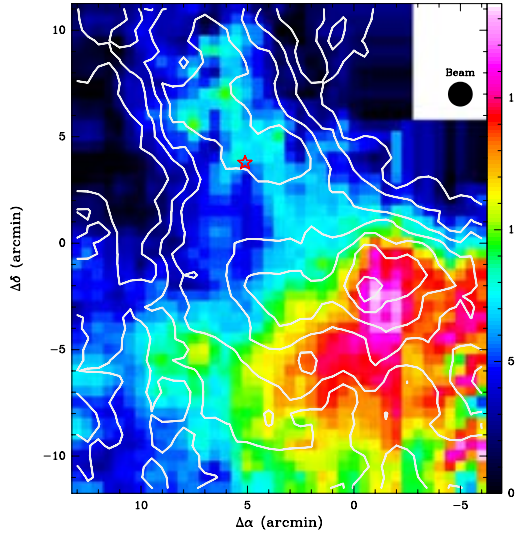

Figure 1. Integrated intensities of [C I] 1-0 emission (color) overlayed with contours of ${ }^{12} \mathrm{CO} 4-3$ (contours) in IC 348. The center of the map is at $\alpha=03^{h} 44^{m} 10^{s}, \delta=$ $32^{\circ} 06^{\prime}$ (J2000). The intensities are integrated from $\mathrm{V}_{L S R} 2 \mathrm{~km} \mathrm{~s}^{-1}$ to $14 \mathrm{~km} \mathrm{~s}^{-1}$. The contour levels are from $10 \mathrm{~K} \mathrm{~km} \mathrm{~s}^{-1}$ to $58 \mathrm{~K} \mathrm{~km} \mathrm{~s}^{-1}$ by a step of $8 \mathrm{~K} \mathrm{~km} \mathrm{~s}^{-1}$. Both data are shown at a common resolution of $70^{\prime \prime}$. The black star denotes the position of HD 281159, a B 5 type star.

IC 348 is one of the most studied young open clusters, which lies at a distance of 320 pc. The [C I] 1-0 emission peaks to the south-west of the mapped region (Fig. 1). The emission extends towards the east and the north-east. The ${ }^{12} \mathrm{CO} 4-3$ intensity also peaks almost at the same position of the [C I] peak, but shows a second peak lying to the north of HD 281159 and elongated along the north-south direction. The features in the ${ }^{12} \mathrm{CO}$ 4-3 map agree quite well with those in ${ }^{12} \mathrm{CO} 3-2$ map (Sun et al. 2006). We attribute the difference in the intensity distributions of $[\mathrm{C} \mathrm{I}]$ and ${ }^{12} \mathrm{CO} 4-3$ to the fact that ${ }^{12} \mathrm{CO}$ 4-3 traces regions of higher temperature, while [C I] traces the embedded PDR surfaces of the molecular clouds with high column density. A plot of the $\mathrm{N}(\mathrm{C}) / \mathrm{N}(\mathrm{CO})$ ratio vs. the $\mathrm{H}_{2}$ column density in IC 348 shows a clear anti-correlation. A linear fit to the data in logarithmic coordinates gives a slope of -0.59 (cf. Fig. 6, Mookerjea et al. 2006).

\section{References}

Mookerjea, B. et al. 2006, A\&\&A 456, 235

Sun, K. et al. 2006, A\&BA 451, 539 